\title{
INVESTIGATING THE EFFECTS OF PROCESS PARAMETERS ON THE FLEXURAL STRENGTH OF 3D PRINTED PLASTICS
}

\author{
Nikolai Beharry $^{1^{*}}$, Boppana V. Chowdary ${ }^{2}$ \\ ${ }^{1,2}$ Faculty of Engineering, The University of the West Indies, Trinidad \\ ${ }^{1}$ Email: nikolai.beharry@my.uwi.edu* \\ ${ }^{2}$ Email: boppana.chowdary@sta.uwi.edu
}

\begin{abstract}
In this paper, the flexural strength of 90\% Recycled Polyethylene Terephthalate (RPET), Polyethylene Terephthalate Glycol (PETG) and Polycarbonate (PC), are investigated with varying layer thickness, infill percentage and raster angle. Across each material, higher infill percentage resulted in higher flexural strength when loaded, whereas raster angle was the least significant. Using a Genetic Algorithm, the maximum flexural strength of each material was determined to be 48.57 MPa for RPET, 44.22 MPa for PETG and $101.54 \mathrm{MPa}$ for the PC samples. Using these results, practical applications in local industries were investigated. The relatively high flexural strength of the RPET indicates great progress in material science towards creating a strong and sustainable 3D Printing filament. However, a cost-benefit analysis performed on the existing market value indicated that in its current state, the price per strength of RPET is heavily outmatched by PETG as a cheaper option and PC as a stronger option. If the process of creating filament from recycled plastics is refined, RPET filament will become cheaper and more desirable for manufacturing. This can lead to diversifying the local economy as a competitive and eco-friendly option to satisfy consumer needs.
\end{abstract}

Keywords: 3D Printing; Flexural Strength; Genetic Algorithm; Recycled Thermoplastic

\section{https://doi.org/10.47412/FBTO9600}

\section{Introduction}

3D Printing of thermoplastics is a widely popular "layer by layer" Additive Manufacturing method used domestically and industrially for product realization and is based on the principle of material extrusion through a nozzle under high heat and pressure. To compete with traditional plastic forming methods, products made with 3D Printing must have comparable or better mechanical properties. Applications range from use in static environments, usually ideal for maintaining the characteristics of the plastics, to environments where they may be subject to dynamic forces or weather conditions that degrade their properties. Thus, the anisotropic strength of the final part must be considered when designing the component and manufacturing process. In 3D Printing, the strength of the product is majorly affected by the chemical makeup of the raw material and the input parameters used during manufacturing setup. Optimal parameters and materials would therefore allow the manufacturer to achieve the best possible product for its intended use. As this is a relatively new manufacturing method, research must be performed to build a baseline database of print parameters for materials, which must be updated as material science develops. This paper seeks to add new empirical data to this database on the strength of 3D Printed products 
by focusing on new materials and variables that lack research. To determine these gaps, one must analyse literature over a large span and determine the trends in the data.

Ahn et al. [1] compared the strengths of 3D Printed and injection molded Acrylonitrile Butadiene Styrene (ABS) samples. They found that the average strengths of the printed samples were lower than the injection molded samples by between $28 \%$ and $35 \%$ with tensile loading, and $10 \%$ to $20 \%$ with compressive loading. As pointed out by Rodríguez, Thomas, and Renaud [2], it is expected that there will be a difference in mechanical properties between 3D Printed parts and nominal material just by the layer by layer nature of the process. In their study, they found the stiffness and strength of 3D Printed ABS samples to be within 0$10 \%$ of the theoretical values determined from standard ABS data. One of the key takeaways from these studies is that there are mechanical limitations to the 3D Printing process due to the fact that layers of material are placed on each other as the extrusion process occurs, leaving tiny air gaps within the structure, which is non-existent in traditional plastic forming.

Print orientation when creating the g-code of an STL file affects the build time and cost, amount and orientation of support materials and dimensional accuracy of the part. Bellini and Güçeri [3] focused their study on the surface quality and dimensional stability to achieve desired mechanical properties for final products and found that the print orientation and the chemical makeup of the filament were key in producing a high tensile strength ABS part. The raster angle, that is, the angle at which material is extruded within the perimeters of the part, is also directly impacted by the print orientation as noted by Hill and Haghi [4] in their analysis of the yield and tensile strengths, elongation, elastic modulus, hardness and density of Polycarbonate (PC) samples. Lee et al. [5] performed compressive tests on ABS samples with varying print orientations and a set raster angle of 45 degrees. One sample set was printed in the axial direction (horizontally), one set transversely (vertically) and one set diagonally on the print bed. They found that the compressive strength of the transverse sample set was around $11.4 \%$ lower than the axial set, and that the diagonally printed samples were the strongest of the three sets. Sood, Ohdar, and Mahapatra [6] took the analysis of the print orientation one step further by applying Particle Swarm Optimization (PSO) and Artificial Neural Network (ANN) techniques to optimize the build orientation, raster angle and other input settings for maximum compressive strength of ABS samples.

Alvarez, Lagos and Aizpun [7] explored trade-off between the tensile strength and impact resistance and the amount of infill percentage for ABS samples. The study showed that $100 \%$ infill produced the strongest sample, but also noted, however, that using an infill percentage between 50 to $98 \%$ was not practical as the print time will be only marginally shorter than $100 \%$ infill but with far poorer mechanical properties. Tensile strength was also investigated for PLA samples by Ezoji, Razavi-Nouri and Rezadoust [8] with varying raster angles, print orientation and layer thickness. They found that as the layer thickness was reduced, the tensile strength increased. Rajpurohit and Dave [9] tested the tensile strength of PLA samples with varying layer thickness and raster properties and concluded that a raster angle of zero degrees and a smaller layer thickness produced samples with the highest tensile strength.

Rahim et al. [10] performed 3-Point Bending tests on ABS specimens to observe the impact of layer thickness, print orientation and raster angle on flexural strength. and found that the most significant factor on build strength was the sample's print orientation. They concluded that the higher the build angle, the smaller the maximum bending stress as they deflected more before fracture, indicating higher ductility. Similarly, Solomon, Rosenthal, Ashkenazi and Stern [11] performed a study on the flexural testing of 3D Printed ABS with varying build orientations and came to similar conclusions. Both groups of researchers varied the print angles at 0,45 and 90 degrees from the $\mathrm{X}$-axis.

From the literature reviewed, tensile strength is the most commonly measured property for 3D Printed samples, as a somewhat benchmark test for part strength. However, in order to maximize the use of 3D Printing products must be able to withstand forces in all directions. This will allow manufacturers to design and create products for a wider range of applications, while maintaining the strength desired for their use. 
There seemed to be a lack of information on the flexural or bending strength of the products, which if more understood, will allow for better design for products susceptible to deformation and buckling under compressive loads. Further, it can be observed that ABS is one of the most studied materials due to its extensive industrial use at a relatively low cost. Thus, this paper seeks to investigate the effects of varying input process parameters during product design, on the maximum flexural strength of 3D Printed thermoplastics. Samples shall be subjected to increasing compressive forces until fracture so that the relevant extension and stress-strain data can be obtained. From this, the input parameters that produce the highest flexural strength can be determined using robust statistical analysis and optimization techniques.

\section{Experimental Setup}

This paper focuses on the influence of three input parameters raster angle, layer thickness and infill \% on the flexural strength of Refill TM Recycled Polyethylene Terephthalate (RPET), eSun TM Polyethylene Terephthalate Glycol (PETG) and Polymaker TM Polycarbonate (PC) samples. The Design of Experiments (DOE) technique used in this paper is the fractional factorial method using a Taguchi L9 Orthogonal Array (OA) for the three input parameters at three levels, that is, low, medium and high, a total of 9 experiments per material. The input levels were determined based on previous similar experimental work. The fractional factorial DOE removes any redundant experiment sets that add little to no value in determining the trends in factor relationships that may be present in other methods like full factorial DOE. Table 1 shows the parameters and values for each level.

Table 1: Process Parameter Levels for Samples

\begin{tabular}{|c|c|c|c|}
\hline Level & $\begin{array}{c}\text { Layer Thickness } \\
(\mathbf{m m})\end{array}$ & Infill (\%) & Raster Angle (deg.) \\
\hline Low & 0.1 & 15 & 0 \\
\hline Medium & 0.2 & 50 & 45 \\
\hline High & 0.3 & 100 & 90 \\
\hline
\end{tabular}

Table 2 shows the levels of the parameters of each sample set for the fractional factorial design.

\begin{tabular}{|c|c|c|c|}
\hline Sample Set & $\begin{array}{c}\text { Layer Thickness } \\
(\mathbf{m m})\end{array}$ & Infill (\%) & Raster Angle (deg.) \\
\hline 1 & Low & Low & Low \\
\hline 2 & Low & Medium & Medium \\
\hline 3 & Low & High & High \\
\hline 4 & Medium & Low & Medium \\
\hline 5 & Medium & Medium & High \\
\hline 6 & Medium & High & Low \\
\hline 7 & High & Low & High \\
\hline
\end{tabular}




\begin{tabular}{|c|c|c|c|}
\hline 8 & High & Medium & Low \\
\hline 9 & High & High & Medium \\
\hline
\end{tabular}

Further, Table 3 shows the process paramters that were kept constant for each sample design to reduce variation.

Table 3: Constant Variables

\begin{tabular}{|c|c|}
\hline Process Parameter & Value \\
\hline Brim Width & $5 \mathrm{~mm}$ \\
\hline Infill Pattern & Rectilinear \\
\hline $\begin{array}{c}\text { Top and Bottom } \\
\text { Horizontal Shells }\end{array}$ & 3 \\
\hline Print Speed & $45 \mathrm{~mm} / \mathrm{s}$ \\
\hline Ambient Temperature & 31 deg. C \\
\hline Nozzle Type & $\begin{array}{c}\text { Brass, } 0.4 \mathrm{~mm} \\
\text { (diameter) }\end{array}$ \\
\hline Filament Size & $1.75 \mathrm{~mm}$ (diameter) \\
\hline
\end{tabular}

Samples were designed using SolidWorks 2017 software according to the ASTM D790 standard with the nominal length adjusted to fit the Tinius Olsen Universal Testing machine. Samples were then printed on a Prusa i3 MK3 3D Printer in a controlled environment. Figure 1 shows the CAD model of the samples with dimensions in $\mathrm{mm}$.
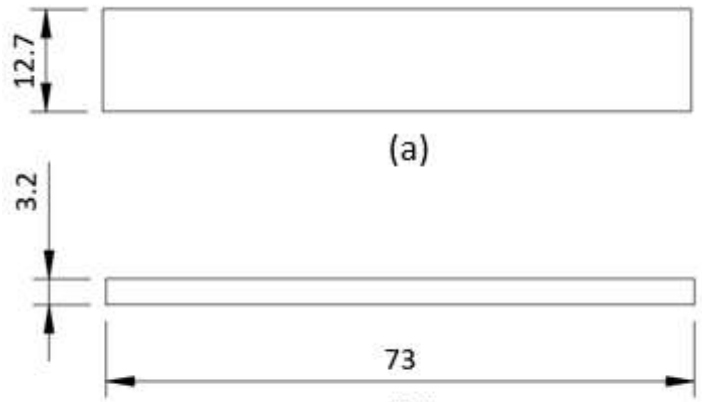

(b)

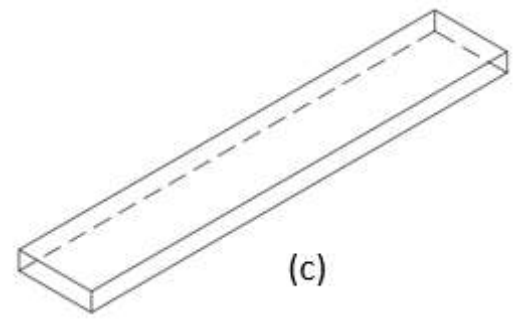

Figure 1: Sample Design; (a) Top View; (b) Side View; (c) Isometric View

After sample creation 3-Point Bending tests were performed on the testing machine according to the ASTM D790 standard on the Tinius Olsen Universal Testing machine. The maximum load and extension experienced by the samples were recorded and analysed using MINITAB 17 software. 
Figure 2 shows the orientation of the samples on the testing machine for the bending tests with dimensions in $\mathrm{mm}$. Each sample is placed evenly on the support beams and the loading pin applies a downward force at a constant rate until fracture.

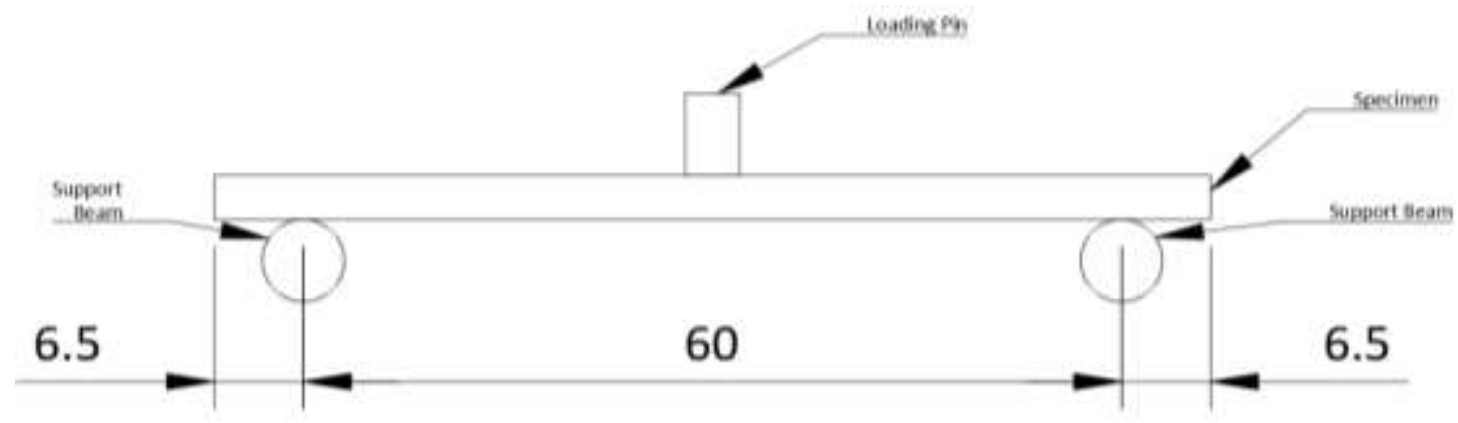

Figure 2: Experimental Setup of Samples on Testing Machine

The equation used to compute the maximum flexural strength of each sample, taken from the ASTM D790 standard, follows:

$$
\sigma_{\mathrm{f}}=(3 \mathrm{PL}) /(2 \mathrm{bd} 2) .
$$

From Eq. (1), P represents a point load on the load-extension curve measured in $\mathrm{N}$, and $\sigma_{\mathrm{f}}$ denotes the flexural strength of the sample at midpoint measured in MPa. The dimensions of the sample, in mm, are represented by $\mathrm{L}$ for support span, $\mathrm{b}$ for the beam width and $\mathrm{d}$ for the beam depth.

\section{Results and Analysis}

\subsection{Data Collected}

The experiments produced the maximum force and extension of the samples under load. These forces indicate the point in which the samples surpass the viscoelastic (recoverable) deformation and begin plastic (permanent) deformation under load. Table 4 shows the average flexural strength calculated using Eq. (1) for the RPET PETG and PC sample strength generated with the 3-Point Bending Tests. Note that this is the average of five replications for each sample set.

Table 4: Average Flexural Strength of Samples

\begin{tabular}{|c|c|c|c|}
\hline $\begin{array}{c}\text { Experiment } \\
\text { No. }\end{array}$ & $\begin{array}{c}\text { Avg. Flexural Strength of } \\
\text { RPET (MPa) }\end{array}$ & $\begin{array}{c}\text { Avg. Flexural Strength of } \\
\text { PETG (MPa) }\end{array}$ & $\begin{array}{c}\text { Avg. Flexural Strength } \\
\text { of PC (MPa) }\end{array}$ \\
\hline 1 & 22.59 & 18.39 & 48.57 \\
\hline 2 & 28.82 & 20.07 & 58.09 \\
\hline 3 & 45.01 & 33.59 & 98.19 \\
\hline 4 & 30.51 & 25.52 & 63.35 \\
\hline 5 & 34.71 & 31.38 & 73.15 \\
\hline 6 & 46.02 & 41.90 & 100.24 \\
\hline
\end{tabular}




\begin{tabular}{|c|c|c|c|}
\hline 7 & 33.69 & 32.30 & 67.60 \\
\hline 8 & 36.60 & 32.30 & 73.86 \\
\hline 9 & 48.57 & 44.22 & 101.54 \\
\hline
\end{tabular}

Evidently for all materials, experiment 9 produced the highest flexural strength value. MINITAB 17 software was used to model the experimental data collected to determine the relationships between the print parameters and the flexural strength. Non-linear regression models were created for each material to define the data collected for each material. Low standard error of regression values were determined, meaning that the models closely represented the data collected. The regression models developed are as follows:

$$
\begin{aligned}
\sigma_{\text {RPET }}= & 12.760+108.10 \mathrm{~A}+0.00881 \mathrm{~B}+0.07057 \mathrm{C}-120.00 \mathrm{~A} * \mathrm{~A} \\
& +0.001976 \mathrm{~B} * \mathrm{~B}-0.2101 \mathrm{~A} * \mathrm{~B}-0.2477 \mathrm{~A} * \mathrm{C} \\
\sigma_{\text {PETG }}= & 4.637+163.75 \mathrm{~A}-0.01089 \mathrm{~B}-0.09881 \mathrm{C}-278.83 \mathrm{~A} * \mathrm{~A} \\
& +0.001671 \mathrm{~B} * \mathrm{~B}+0.000843 \mathrm{C} * \mathrm{C}+0.2015 \mathrm{~A} * \mathrm{C} \\
\sigma_{\mathrm{PC}}= & 17.65+358.7 \mathrm{~A}+0.0615 \mathrm{~B}-0.0559 \mathrm{C}-584.0 \mathrm{~A} * \mathrm{~A} \\
& +0.004575 \mathrm{~B} * \mathrm{~B}-0.8896 \mathrm{~A} * \mathrm{~B}+0.001393 \mathrm{~B} * \mathrm{C}
\end{aligned}
$$

Eq. (2), Eq. (3) and Eq. (4) represents the flexural strength models for RPET, PETG and PC, respectively. " $\mathrm{A}$ " is the Layer Thickness, " $\mathrm{B}$ " is the Infill \% and "C" is the Raster Angle. It can be observed that the equations weigh individual terms and interactions of different levels by assigning constants, where the larger constant indicates a larger effect on the flexural strength of the material.

The residual and main effect plots among the three materials were similar in that the model followed the assumptions of normality and non-discernible patterns. Further, by the interaction and surface plots for the three materials, it was apparent that the infill percentage had the highest impact on the flexural strength, where the higher the percent, the stronger the sample. This is similar to the results found in papers such as Johnson and French [12] where the infill had a very high impact on strength, elastic modulus, elongation and failure modes of 3D Printed samples. Further analysis showed that the raster angle had the least significant impact on the three materials.

\subsection{GA Optimization}

Following this data analysis, the optimal process parameters was determined for maximum strength by mapping the non-linear regression models developed to the GA optimization technique using MATLAB. The aim of this optimization is to enhance product design by finding the best combination of print settings to maximize the flexural strength of RPET, PETG and PC. Validation samples were printed using the optimal settings and the actual flexural strength was compared to that estimated by the GA. This can be observed in Table 5.

Table 5: Comparison between Estimated and Actual Flexural Strength of Validation Samples

\begin{tabular}{|c|c|c|c|c|c|}
\hline Material & $\begin{array}{c}\text { Layer } \\
\text { Thickness } \\
(\mathbf{m m})\end{array}$ & Infill (\%) & $\begin{array}{c}\text { Raster Angle } \\
\text { (deg.) }\end{array}$ & $\begin{array}{c}\text { Estimated Flex. } \\
\text { Stress (MPa) }\end{array}$ & $\begin{array}{c}\text { Actual Flex. } \\
\text { Stress (MPa) }\end{array}$ \\
\hline RPET & 0.3 & 100 & 0 & 48.72 & 48.57 \\
\hline
\end{tabular}




\begin{tabular}{|c|c|c|c|c|c|}
\hline PETG & 0.3 & 100 & 90 & 47.69 & 41.90 \\
\hline PC & 0.23 & 100 & 90 & 108.20 & 97.26 \\
\hline
\end{tabular}

\section{Discussion}

\subsection{GA Analysis}

From Table 4, the actual stress values from the validation experiments were lower than that predicted by the GA. The RPET sample was the closest in relation, being only $0.31 \%$ lower than the predicted value. However, the PETG and PC samples experienced maximum flexural strengths that were $12.1 \%$ and $10.1 \%$ lower than the estimated GA values, respectively.

Since the non-linear regression equations for the PETG and PC samples seemed to closely fit the data collected from the initial Design of Experiments this large deviation may be attributed to the GA's inability to account for controllable or uncontrollable factors that impact the sample strength. There may have been some unexpected interactions between print settings not considered in the DOE that also affected the flexural strength of the samples, which had a high significance when creating the validation samples. Theoretically, for such a simple optimization, the GA should have no issue in finding the best value. However, there may have been some premature convergence to local optima which caused the GA to stop simulating before a true optimal value was found. This could have been controlled through extensive work in selecting the input settings for the GA to suit the data being examined. Further, while every effort was made to ensure the environment where the samples were being created was controlled and constant, some varying noise factors may have been present.

As this research area of 3D Printing is relatively new and still being developed, there is little publicly accessible benchmark data on the flexural strength and optimal print settings of the materials being tested. This makes it difficult to get a conclusive idea on the statistical validity as there are little globally recognized standards in flexural strength analysis of every type and branding of 3D Printer materials with which one can compare. Nonetheless, the flexural strength of the $90 \%$ recycled-bottle RPET sample determined in this paper can be considered as a strong reference or benchmark for future researchers due to the high statistical validity of the regression and optimization models.

\subsection{Design Guidelines}

From this analysis, the recommended print settings for maximum achievable flexural strength are those that follow the GA for the RPET, and those that follow Experiment 9 for PETG and PC. Table 6 lists the settings and sample strength that is to be expected.

Table 6: Recommended Print Settings

\begin{tabular}{|c|c|c|c|c|}
\hline Material & $\begin{array}{c}\text { Layer } \\
\text { Thickness } \\
(\mathbf{m m})\end{array}$ & Infill (\%) & $\begin{array}{c}\text { Raster Angle } \\
(\mathbf{d e g})\end{array}$ & $\begin{array}{c}\text { Expected Maximum } \\
\text { Flexural strength (MPa) }\end{array}$ \\
\hline RPET & 0.3 & 100 & 0 & 48.57 \\
\hline PETG & 0.3 & 100 & 45 & 44.22 \\
\hline PC & 0.3 & 100 & 45 & 101.54 \\
\hline
\end{tabular}




\subsection{Cost Benefit Analysis}

From the data collected, the managerial implications and industrial applications of the materials can be determined using a Cost-Benefit Analysis. Figure 3 shows a cost versus strength analysis for the three materials which gives a good overview on the value of each material.

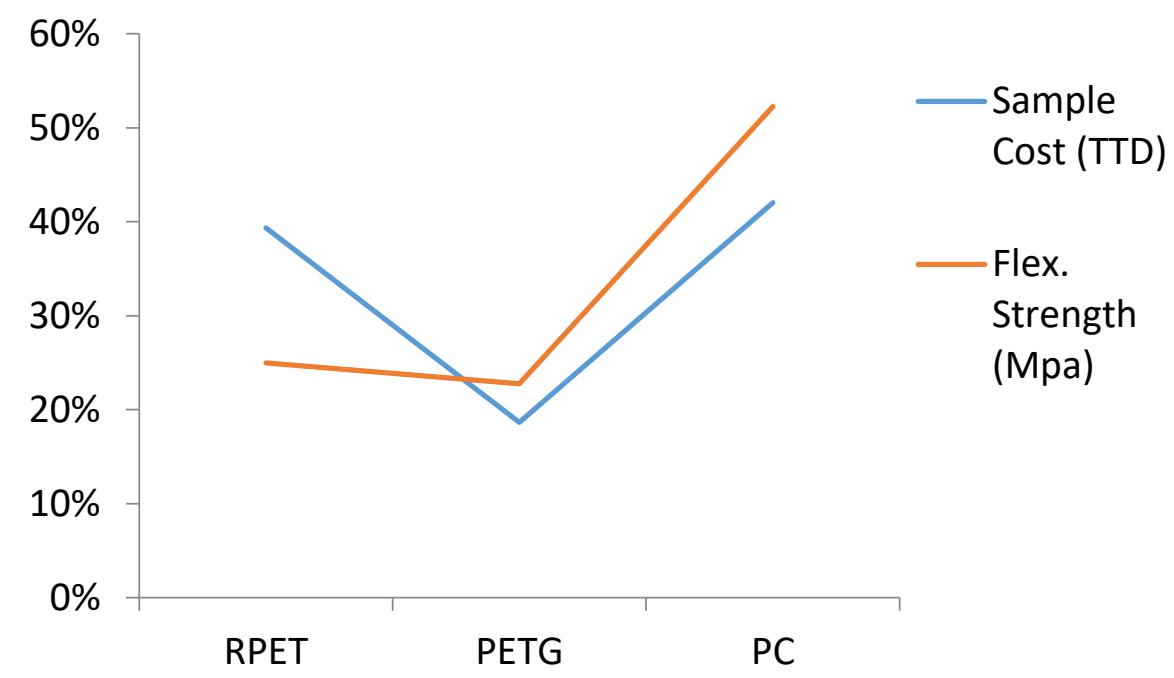

Figure 3: Samples Cost (TTD) vs. Flexural Strength (MPa) of Samples

Clearly, PC has the highest strength of all the samples. It also has, however, the highest cost. PC can be used where high compressive loads and rough external factors are expected such as protective gear, greenhouses, exterior lighting fixtures and furniture. The high maximum flexural strength indicates that it will be sufficient for applications like chairs and tables where the design and size of legs can be customized to create a rigid product. Due to the relatively expensive cost of PC investment into using this material for a final product must be justified by the strength required in product design.

PETG is a more economical choice as it is much cheaper than PC but still relatively strong and easy to print. PETG can be used for internal car parts like fan grills and educational tools as it is cheaper but still extremely strong and chemical and heat resistant. Other applications for PETG include food and beverage industry for impact resistant storage containers that can be adequately sterilized. RPET applications are similar to the PETG as their chemical make-ups and evidently, their mechanical properties are closely related. The $90 \%$ recycled-bottle RPET studied in this paper indicates that the area of recycled plastics is heading in the direction of competitively strong products. Recycled plastics are being made into waste sorting bins, pallet racks, clothing and plant pots etc. using traditional manufacturing methods. However, as the 3D Printing process is much different than traditional methods, optimal settings and suitable product design must be chosen to compliment the additive nature of the process. Other uses of RPET include ecofriendly textiles, rugs, sleeping bags and clothing. The current cost of production may be high with RPET, but custom, niche products like shoes can drive the selling price to an acceptable level for the use of the RPET filament. RPET from plastic bottles have traditionally been reused to manufacture new bottles. However, with the 3D Printing process as it is currently, tiny air gaps between material layers make storing of liquid a difficult process. Often further additions such as internal wall coatings are required to stop liquid from seeping through the layers which increases the complexity and cost of the manufacturing process. Thus, to make the most of the 3D Printing, product choice and designs must consider the manufacturing process and limitations that come with it. By making suitable trade-offs, products with high customizability and adequate mechanical properties can be manufactured. 


\section{Conclusions}

This paper investigated the effects of three 3D Printing process parameters (print settings), namely, the layer thickness (mm), infill percentage (\%) and raster angle (degrees) on the flexural strength of Recycled Polyethylene Terephthalate (RPET), Polyethylene Terephthalate Glycol (PETG) and Polycarbonate (PC) samples. ANOVA and RSM were applied to examine and understand the empirical data collected from the fractional factorial DOE. A GA was then applied to seek the optimal parameters for obtaining the highest flexural strength. The statistical validity of the optimized validation sample for the RPET was acceptable, though for the PETG and PC samples, external and uncontrollable noise factors resulted in larger margins of error. The maximum flexural strength of each material was determined to be $48.57 \mathrm{MPa}$ for RPET, 44.22 MPa for PETG and 101.54 MPa for PC samples. The print settings to achieve these mechanical properties can be observed in Table 6. Designers can follow these print settings to maximize the strength of their products.

The managerial implications of the experiments and optimization were investigated, and a brief cost-benefit analysis was completed to analyse the value of each material. It was determined that, in its current state, RPET might not be the most practical for medium-large scale manufacturing due to the high cost per flexural strength ratio. Several practical applications of the materials were outlined in the fields of FoodProcessing and Furniture Manufacturing and feasibility analyses were performed.

The empirical data collected and analysed in this paper should aid designers in choosing the most suitable process parameters for the intended product requirements. 3D Printing and Additive Manufacturing can certainly become a more competitive option to traditional plastic forming methods with adequate and aggressive investment in material and process research and development. Some of the areas that researchers conducting similar studies can consider to enhance the quality of their research include:

1. Using a DOE technique that considers a wider range of parameter interactions, such as Full Factorial, especially if more than three print settings are being investigated.

2. Conducting qualitative research through questionnaires, surveys etc. will further enhance the understanding the expected trade-offs between mechanical properties, and manufacturing cost and efficiency. This will allow for a more detailed cost-benefit analysis.

3. Comparing the properties of 3D Printed samples to those made from the same material but traditional plastic forming techniques. This would be a very compelling way to market the viability of 3D Printing as a competitive manufacturing process.

4. Researching and refining the manufacturing process of recycled filament.

5. Exploring and utilizing combined or different optimization techniques to understand the difference in statistical variation between two methods like GA versus PSO.

\section{References}

[1] S. Ahn, M. Montero, D. Odell, S. Roundy, P. Wright. 2002. Anisotropic Material Properties of Fused Deposition Modeling ABS. In Rapid Prototyping Journal 8 (4). https://search-proquestcom.ezproxygateway.sastudents.uwi.tt/docview/214025240? accountid=45039.

[2] J. Rodríguez, J. Thomas, J. Renaud. 2003. Mechanical Behavior of Acrylonitrile Butadiene Styrene Fused Deposition Materials Modeling. In Rapid Prototyping Journal 9 (4): 219-230. https://doi.org/https://doi.org/10.1108/13552540310489604.

[3] A. Bellini, S. Güçeri. 2003. Mechanical Characterization of Parts Fabricated Using Fused Deposition $\begin{array}{lllllll}\text { Modeling. } & \text { In } & \text { Rapid } & \text { Prototyping } & \text { Journal } & 9 & \text { (4): }\end{array}$ https://doi.org/https://doi.org/10.1108/13552540310489631. 
[4] N. Hill, M. Haghi. 2014. Deposition Direction-Dependent Failure Criteria for Fused Deposition Modeling Polycarbonate. In Rapid Prototyping Journal 20 (3): 221-227. https://doi.org/10.1108/RPJ-042013-0039.

[5] C. Lee, S.G. Kim, H. Kim, and S. Ahn. 2007. Measurement of Anisotropic Compressive Strength of Rapid Prototyping Parts. In Journal of Materials Processing Technology 187 (627-630). https://doi.org/10.1016/j.jmatprotec.2006.11.095.

[6] K. Sood, R. Ohdar, S. Mahapatra. 2012. Experimental Investigation and Empirical Modelling of FDM Process for Compressive Strength Improvement. In Journal of Advanced Research 3 (1): 81-90. https://doi.org/https://doi.org/10.1016/j.jare.2011.05.001.

[7] K. Álvarez, R. Lagos, M. Aizpun. 2016. Investigating the Influence of Infill Percentage on the Mechanical Properties of Fused Deposition Modelled ABS Parts. In Ingeniería e Investigación 36 (3). https://doi.org/10.15446/ing.investig.v36n3.56610.

[8] M. Ezoji, M. Razavi-Nouri, A. Rezadoust. 2017. Optimization of Processing Parameters in 3D-Printing of Polylactic Acid by Fused Deposition Modeling Method. In Iran. J. Polym. Sci. Technology 30 (2): 115126. https://doi.org/10.22063/jipst.2017.1481.

[9] S. Rajpurohit, H. Dave. 2018. Effect of process parameters on tensile strength of FDM printed PLA part. In Rapid Prototyping Journal 24 (8): 1317-1324. https://doi.org/10.1108/rpj-06-2017-0134.

[10] T. Rahim, A. Abdullah, H. Akil, D. Mohamad, Z. Rajion. 2017. The Improvement of Mechanical and Thermal Properties of Polyamide 12 3D Printed Parts by Fused Deposition Modelling. In Express Polymer Letters 11 (12): 963-982. https://doi.org/10.3144/expresspolymlett.2017.92.

[11] A. Solomon, Y. Rosenthal, D. Ashkenazi, A. Stern. 2018. Structure and Mechanical Behavious of Additive Manufactured Fused Deposition Modeling ABS. In the Annals of Dunarea De Jos University of Galati Fascicle XII - Welding Equipment and Technology 29: 47-56.

[12] G. Johnson, J. French. 2018. Evaluation of Infill Effect on Mechanical Properties of Consumer 3D Printing Materials. In Advances in Technology Innovation 3 (4): 179-184. 